\title{
Novel Fishnet-like Chiral Metamaterial Structure with Negative Refractive Index and Low Losses
}

\author{
Oscar Fernández, Álvaro Gómez \\ and Angel Vegas \\ Dpto. Ingeniería de Comunicaciones \\ Universidad de Cantabria \\ E39005 Santander, Spain \\ fernanos@unican.es, \\ gomezal@unican.es, \\ vegasa@unican.es
}

\author{
Gregorio J. Molina-Cuberos \\ Dpto. Electromagnetismo y \\ Electrónica \\ Universidad de Murcia \\ E30100 Murcia, Spain \\ gregomc@um.es
}

\author{
Angel. J. García-Collado \\ Dpto. Ciencias Politécnicas \\ Universidad Católica de San Antonio \\ E30107 Murcia, Spain \\ ajgarcia@ucam.edu
}

\begin{abstract}
In this work a composed chiral metamaterial structure constituted by the combination of a composite chiral metamaterial and a modified fishnet structure is presented and numerically analyzed. This new fishnet-like chiral metamaterial features two wide frequency bands wherein the refractive indices are negative and exhibits lower losses than its constituents do.
\end{abstract} index

Keywords_chiral; fishnet; metamaterial; low loss; refractive

\section{INTRODUCTION}

Chiral metamaterials (CMM) provide an alternative route to negative refraction [1]. Thanks to their chirality parameter, CMMs can exhibit negative refractive index, $n_{ \pm}$, for the right$(+)$ or left-handed $(-)$ circularly polarized eigenwaves. A drawback of these structures is the high losses they may present. A way of partially decreasing these losses is provided by using composite CMMs (CCMM) [2].

In a previous work [3] the authors presented a CCMM structure with negative refractive index for one eigenwave, $\left|n_{+}\right|<0$, and high Figure of Merit, $\operatorname{FoM}\left(n_{+}\right)=\left|\operatorname{Re}\left(n_{+}\right) / \operatorname{Im}\left(n_{+}\right)\right|$. With the aim of going a step further on the losses reduction, an enhanced evolution of the aforementioned mentioned structure is presented in this communication. This new design, called fishnet-like chiral metamaterial (FCMM), displays $\mathrm{C}_{4}$ symmetry and combines the CCMM of [3] with a low losses fishnet arrangement [4]-[5]. This novel combination improves the response of [3] by providing negative refractive indices for each eigenwave along two wider frequency bands $(0.8$ and $0.9 \mathrm{GHz})$ and also a higher FoM, greater than 50 in the $60 \%$ of each frequency band.

\section{FISHNET LIKE CHIRAL METAMATERIAL}

Fig. 1 shows the CCMM and the modified fishnet structures used to build the proposed FCMM, which is also presented in the figure. The selected substrate is Rogers RO4003C, with dielectric constant $\varepsilon_{r}=3.38$, $\tan \delta=0.0027$, thickness $d=1.52$ $\mathrm{mm}$ and a copper cladding of $35 \mu \mathrm{m}$. The geometrical parameters of the structure are displayed in the figure caption. The FCMM structure has been characterized by means of the

This work has been partially supported by the Spanish Governmen MINECO through the ERDF co-funded Research Projects TEC2014-55463C3-1-P and TEC2014-55463-C3-3-P. classical parameter retrieval algorithm proposed in [6]. The transmission and reflection coefficients for linearly polarized incident waves have been obtained through numerical simulations using the Finite Differences Time Domain engine of the Keysight EMPro 3D EM software ${ }^{\circledR}$. For all simulations, an $e^{j w t}$ time dependence has been considered.

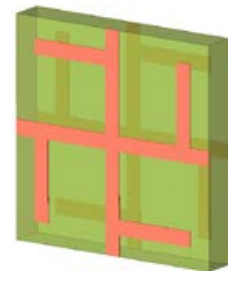

(a)

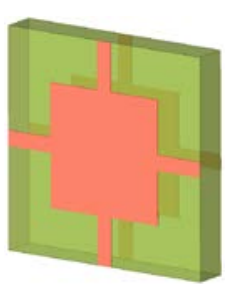

(b)

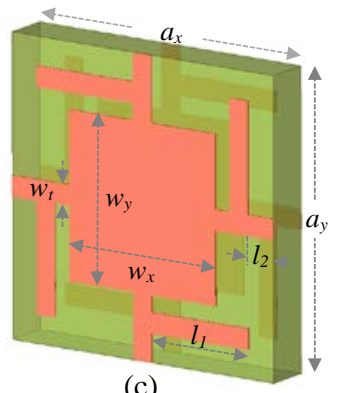

(c)
Fig. 1. (a) CCMM [3] and (b) modified fishnet structures used to create the proposed FCMM (c). Dimensions: $l_{1}=3.7 \mathrm{~mm}, l_{2}=0.95 \mathrm{~mm}, w_{t}=0.7 \mathrm{~mm}$, $w_{x}=w_{y}=5.6 \mathrm{~mm} a_{x}=a_{y}=10 \mathrm{~mm}$.

\section{A. Analysis of FCMM constituents}

Prior to the analysis of the fishnet-like CMM structure, the CCMM and the modified fishnet structures are briefly examined paying special attention to the variation of the transmission coefficients and the refractive indices versus frequency shown in Fig. 2 and Fig. 3, respectively. The chiral behavior of the CCMM produces a co-polar transmission with two dips at 10 and $13.4 \mathrm{GHz}$ and a cross-polar transmission with a peak at 10.8 $\mathrm{GHz}$, Fig. 2. Furthermore, the refractive indices of both eigenwaves are negative in a frequency band above each of the resonance frequencies, Fig. 3a. However, only $n_{+}$presents a frequency band, between 10.9 and $11.5 \mathrm{GHz}$, wherein $n^{\prime}+<0$ and $n$ " is close to zero. In this range the maximum value of $\operatorname{FoM}\left(n_{+}\right)$is 40 .

The fishnet pattern is non-chiral and, as the CCMM does, displays $\mathrm{C}_{4}$ symmetry. This structure exhibits a magnetic resonance at $14.5 \mathrm{GHz}$, which produces a dip in the co-polar transmission coefficient (Fig. 2), and a peak with positive value in the refractive index (Fig. 3b). 


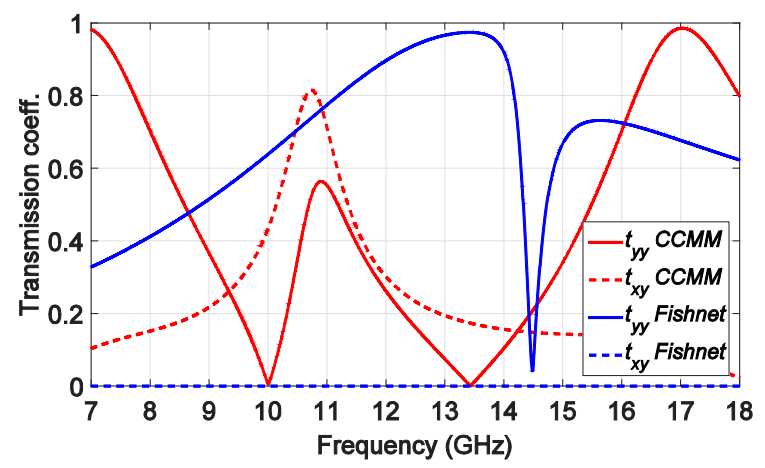

Fig. 2. Transmission coefficients of the chiral and the fishnet metamaterial structures.
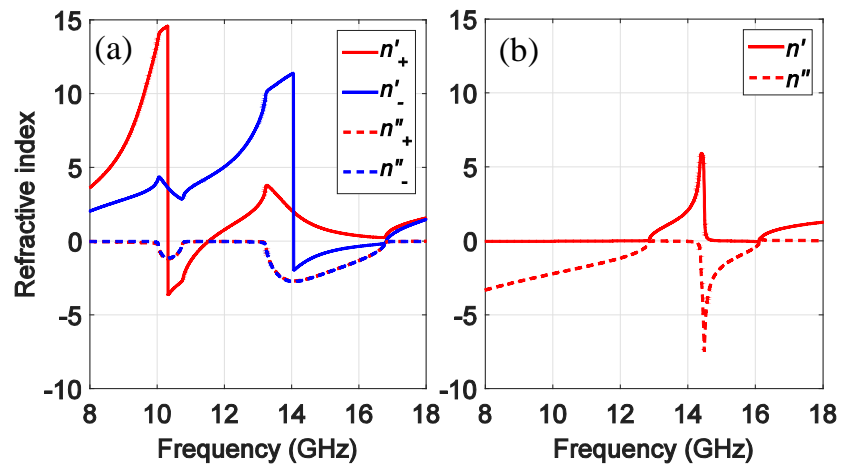

Fig. 3. Refractive indices of the (a) CCMM and (b) the fishnet metamaterials.

\section{B. Analysis of the FCMM structure}

Once each of the components of the FCMM has been analyzed separately, the whole FCMM structure, Fig. 1c, is then examined. On inspecting the variation of the transmission coefficients versus frequency, Fig. 4, it can be observed that, for frequencies lower than $14 \mathrm{GHz}$ the transmission features are very similar to those exhibited by the CCMM structure (Fig. 2). However, for higher frequencies the transmission performance is quite different, presenting the FCMM a chiral behavior that the CCMM does not, which is evident due to the displayed high cross-polar transmission, with two peaks at 14.85 and $16.5 \mathrm{GHz}$. Moreover, the co-polar transmission shows a dip at $15.6 \mathrm{GHz}$.

Finally, the refractive indices $n_{ \pm}$for both eigenwaves (see Fig. 5) presents three resonances, two of them on $n_{+}$at 10.95 and 13.7 GHz, marked in Fig. 5 as A and B respectively, and the third one on $n_{-}$at $17.1 \mathrm{GHz}$, labeled as C. It is worth of mention that the real part of the refractive indices is negative in a finite frequency band just above each of the resonance frequencies. The bandwidths (BW) with $n^{\prime}+<0$ above resonance $\mathrm{A}$ and $\mathrm{B}$ are $\mathrm{BW}_{\mathrm{A}}=0.9 \mathrm{GHz}$ and $\mathrm{BW}_{\mathrm{B}}=1.8 \mathrm{GHz}$ while above resonance $\mathrm{C}$ $n_{-}^{\prime}<0$ in $\mathrm{BW}_{\mathrm{C}}=0.8 \mathrm{GHz}$.

Although $\mathrm{BW}_{\mathrm{B}}$ is very wide, it only presents a narrow region, between 14.7 and $15.3 \mathrm{GHz}$, with low losses, i.e., $n$ "+ near zero. In this band, the maximum $\operatorname{FoM}\left(n_{+}\right)$is only 22 . However in $\mathrm{BW}_{\mathrm{A}}$ and $\mathrm{BW},\left|{ }_{\mathrm{C}}{ }_{+}\right|$is very close to 0 in the whole subband, even near the resonances. With these refractive indices, $\operatorname{FoM}\left(n_{ \pm}\right)$is high, greater than 50 in more than the $60 \%$ of each frequency band. Consequently, these two frequency bands are more recommendable for taking advance of the negative refraction than $\mathrm{BW}_{\mathrm{B}}$.

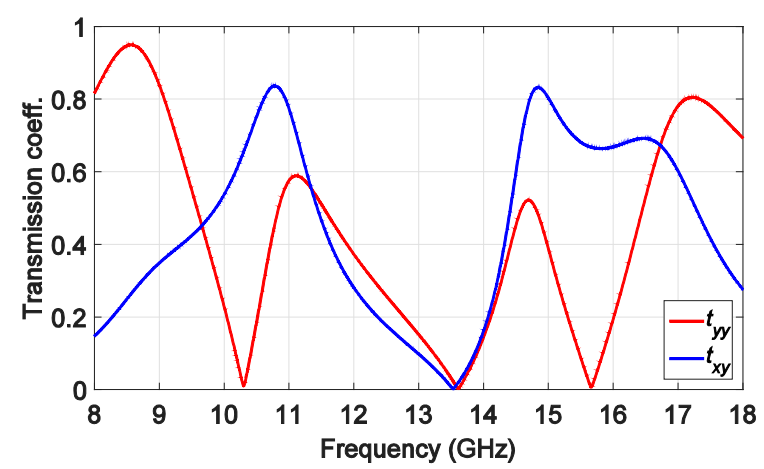

Fig. 4. Transmission coefficients of the FCMM structure of Fig. 1c.

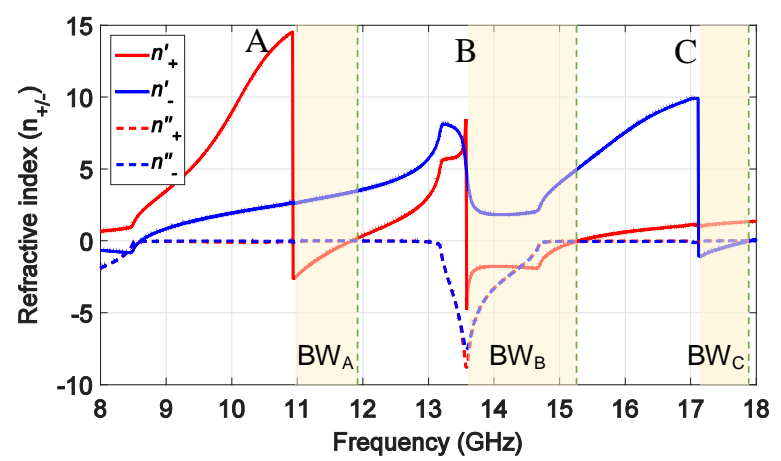

Fig. 5. Refractive indices of the fishnet-like Chiral Metamaterial structure.

\section{CONCLUSIONS}

In this work, it has been presented and analyzed a new chiral metamaterial structure constituted by merging the combination of a composite chiral metamaterial and a modified fishnet structure that enhances the features of the original composite chiral metamaterial.

This new chiral metamaterial structure exhibits negative refractive indices in three frequency bands. Two of these bands present low losses and $\operatorname{FoM}\left(n_{ \pm}\right)$higher than 50 in a bandwidth greater than $0.5 \mathrm{GHz}$ in each band.

\section{REFERENCES}

[1] J. B. Pendry, “A chiral route to negative refraction,” Science, vol. 306, no. 5700, pp. 1353-1355, 2004.

[2] Z. F. Li, K. B. Alici, H. Caglayan, M. Kafesaki, C. M. Soukoulis, and E. Ozbay, "Composite chiral metamaterials with negative refractive index and high values of the figure of merit," Opt. Express, vol. 20, pp. 61466156, 2012.

[3] O. Fernández, Á. Gómez, A. Vegas, G. J. Molina-Cuberos, and A. J. García-Collado, "Losses reduction in composite chiral metamaterials," The 36th PIERS in Prague, Czech Republic, 6-9 July, 2015.

[4] J. Zhou, T. Koschny, and C. M. Soukoulis, "An efficient way to reduce losses of left-handed metamaterials,” Opt. Express, vol. 16, pp. 1114711152, 2008.

[5] M. Kafesaki, I. Tsiapa, N. Katsarakis, Th. Koschny, C. M. Soukoulis, and E. N. Economou, "Left-handed metamaterials: The fishnet structure and its variations," Physical Review B, vol. 75, 235114, 2007.

[6] R. Zhao, T. Koschny, and C. M. Soukoulis, "Chiral metamaterials: retrieval of the effective parameters with and without substrate," Opt. Express, vol. 18, no. 14, pp. 14553-14567, 2010. 